\title{
An ANN-Based Backward Prediction Model for Reliable Bridge Management System Implementations Using Limited Inspection Records - Case Studies
}

\author{
Jaeho Lee \\ Research Fellow \\ Griffith School of \\ Engineering, Gold Coast \\ Campus, Griffith \\ University, QLD 4222, \\ Australia \\ J.lee@griffith.edu.au
}

\author{
Hong Guan \\ Senior Lecturer \\ Griffith School of \\ Engineering, Gold Coast \\ Campus, Griffith \\ University, QLD 4222, \\ Australia \\ H.Guan@griffith.edu.au
}

\author{
Yew-Chaye Loo \\ Director of International \\ and Professional Liaison \\ for SEET Group and \\ Foundation Professor of \\ Griffith School of \\ Engineering, Gold Coast \\ Campus, Griffith \\ University, QLD 4222, \\ Australia \\ Y.Loo@griffith.edu.au
}

\author{
Michael Blumenstein \\ Head, School of \\ Information and \\ Communication Tech- \\ nology, Gold Coast \\ Campus, Griffith \\ University, QLD 4222, \\ Australia \\ M.Blumenstein@griffith.edu.au
}

\section{Summary}

Computer-aided Bridge Management Systems (BMSs) as Decision Support Systems (DSSs) for an effective bridge asset management are used to establish the feasible bridge maintenance, repair and rehabilitation (MR\&R) strategies which ensure an adequate level of safety at the lowest possible bridge life-cycle cost. To achieve this goal, keeping up-to-date bridge condition ratings are crucial for a BMS software package. Although most bridge agencies in the past have conducted inspections and maintenance, the form of such bridge inspection records is dissimilar to those required by BMSs. These data inconsistencies inevitably slow down the BMS implementations. This paper presents an Artificial Neural Network (ANN) based prediction model, called the Backward Prediction Model (BPM), for generating unavailable years of historical bridge condition ratings using very limited existing inspection records. The BPM employed historical non-bridge datasets such as traffic volumes, populations and climates, to establish correlations with the existing bridge condition ratings from the very limited bridge inspection records. Such correlations can help fill the condition rating gaps required for an effective and accurate BMS implementation. This paper covers a brief description of the BPM methodology and presents nine case studies. The outcome of this study can help establish a comprehensive condition rating database, which will in turn assist to predict reliable future bridge depreciations.

Keywords: Bridge Management System (BMS); Backward Prediction Model (BPM); Bridge condition ratings; Artificial Neural Networks (ANNs); Maintenance, Repair and Rehabilitation (MR\&R).

\section{Introduction}

Maintaining bridges at right time are momentous to those service authorities for the all users, because most infrastructure facilities were planned, designed, constructed, operated and modified or rehabilitated under uncertain and risky conditions [1]. For an effective bridge asset management, computer-aided Bridge Management Systems (BMSs) have been developed in the early 1990s. The BMSs have already become a common tool as Decision Support Systems (DSSs) to extend bridge life cycle with minimum costs. Proper operation of BMSs requires massive amount of structural/non-structural information, i.e. inventory, inspection, maintenance records, cost related 
records, and traffic survey and accident report. Among the information required, condition ratings obtained from routine bridge inspections are very important to keep the system up-to-date. The condition ratings obtained are mainly used to evaluate current bridge condition ratings and calculate future bridge depreciations in the system. The evaluation and calculation results are also significant for many other calculations for future bridge needs.

There are a number of shortcomings in using and implementing BMS properly from the perspective of bridge authorities. They are: (1) maximum number of biennial condition rating records are only 6 to 7 sets because BMS commercial packages have only become available to bridge authorities in the early 1990s; (2) The existing limited data do not contain much condition rating variances over a short period time; (3) $60 \%$ of BMS analytical processes rely heavily on the periodic bridge inspection results [2]. These shortcomings are very common problem to all bridge authorities and they seriously affect the prediction of future bridge deterioration of predictions. In relation to the lack of historical bridge condition ratings, most BMS software packages currently in operation in Australia and worldwide suffer the following problems [3-6]: (1) The predicted bridge condition ratings do not acceptably match the real situation; (2) Interactive deterioration mechanism effects between or among structural elements are ignored; (3) One of the assumptions made in most deterioration models is that future conditions are dependent only on the current structural conditions because the past condition ratings are unavailable; (4) Among BMS data requirements, the amount of time-dependent bridge datasets from periodic bridge inspections for a BMS update is very limited; and (5) Bridge condition rating variances in the small number of historical datasets inevitably lead to unreliable structural performance predictions by current deterioration models in BMSs. In view of the above, current BMS outcomes can hardly be expected to be reliable. Thus, the proposed research is very important to improve the prediction quality of deterioration models and thereby, the reliability of overall BMS outcomes.

Extensive research has been conducted to improve the reliability of BMS outcomes. For example, numerous bridge condition rating and deterioration models have been developed to reliably determine the bridge life cycle for the remaining years of use for establishing MR\&R strategies [712]. Time series approaches including Regression, Markov models, Bayesian methods, Fuzzy techniques, Genetic Algorithms, Case Based Reasoning, Artificial Neural Network models and Markov Decision Processes (MDP) have been used in BMS software as part of their deterioration modules. None of these methods is able to generate missing datasets and all suffer inaccurate prediction of long-term bridge performance as a result. Note in general that for these conventional techniques to provide reliable predictions, the percentage of missing data in an entire dataset must be $5 \%$ or less [13]. Despite many previous research achievements, such fundamental problems as the inadequate number of bridge inspection records for BMS input requirements still remains an issue to be overcome. Many researchers and infrastructure asset management practitioners also have recognised that deterioration of infrastructure facilities is not deterministic [14]. Thus, current BMS outcomes are still not practically reliable. To address such research problem, this paper presents the establishment of comprehensive condition rating datasets as part of the effort contributing to a reliable prediction of future bridge depreciations.

\section{Backward Prediction Model}

The proposed methodology has been developed using the National Bridge Inventory (NBI) data due to the limitation of BMS' bridge condition ratings at the disposal of the BMS. In the BPM methodology, the actual BMS' condition rating inputs are tested on same bridge to demonstrate the contribution of the BPM on BMS [15].

\subsection{Mechanism of the BPM}

Fig. 1 schematically describes the mechanism of the BPM (Backward Prediction Model). It illustrates the main function of the ANN technique in establishing the correlation between the existing condition rating datasets (from year $m$ to year $m+n$ ) and the corresponding years' nonbridge factors such as traffic volume, population growth and climatic conditions. The non-bridge factors directly and indirectly affect the variation of the bridge conditions thereby the deterioration rate. The relationships established using neural networks are then applied to the non-bridge factors (for year 0 to year $\mathrm{m}$ ) to generate the missing bridge condition ratings (for the same year 0 to year $\mathrm{m})$. Thus, the non-bridge factors in conjunction with the ANN technique can produce the historical 


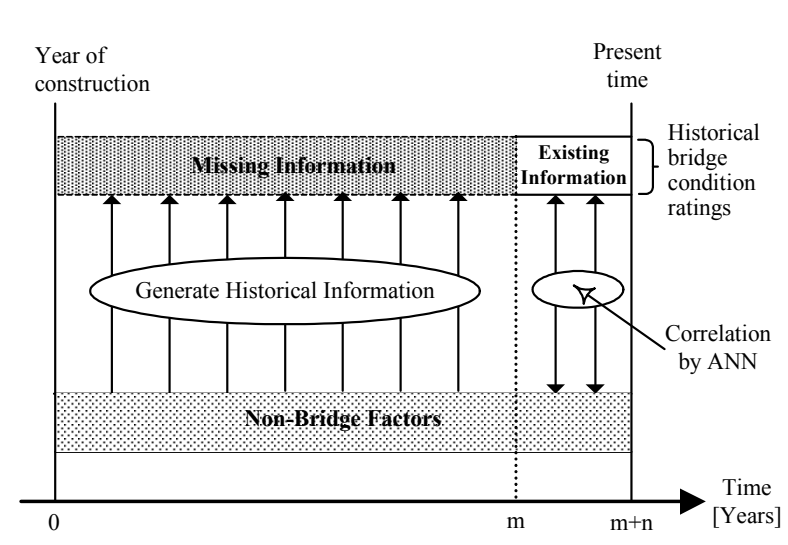

Fig. 1: Mechanism of the BPM trends that inform the current condition ratings. The training algorithm and transfer function used in the BPM are respectively the back-propagation algorithm and the log-sigmoid function. The input layer may have such non-bridge factors as the number of vehicles, population growth and climatic conditions and more. This information is used to train the ANN to determine the correlation with currently available bridge condition rating data in the output layer. Two validation methods have been established to measure the BPM prediction accuracy. The BPM has been tested using two different types of bridge condition rating datasets - the National Bridge Inventory (NBI) and BMS condition rating inputs - for the same bridge provided by the Maryland Department of Transport (DoT), USA.

\subsection{Establishment of the BPM methodology}

\subsubsection{Backward predictions and comparisons}

The five occasions of NBI (from 1996 in 2-year increments to 2004) use the ANN training inputs in the BPM, which generates historical condition ratings from 1968 to 1994 with two-year intervals. The assumed condition rating (excellent condition) at year zero (1966) of the bridge has also been used. The remaining years (1968-1994 with 2 year increments) of historical condition ratings are generated. The results of a BPM (1968-1994) have shown reliable back-prediction performance. The generated historical condition ratings are compared with the existing NBI datasets (1968-1994: 14 missing inspection records) to measure its prediction errors. The BPM generated $73.7 \%$ of the missing years' condition ratings using $26.3 \%$ of the available bridge condition ratings. That is: the missing/entire inspection records $=14 / 19$; the existing/entire inspection records $=4 / 19$. Most artificially-generated historical condition ratings are obtained within a maximum prediction error allowance of $10 \%$ for all bridge components. The prediction differences with the actual NBI (19681994) of the deck, superstructure and substructure are $6.68 \%, 6.61 \%$ and $7.52 \%$, respectively. The results of the backward predictions are validated by comparing them with existing historical condition ratings (Test \#1). However, the actual element-level condition ratings for BMS inputs only have a small number of datasets and are not applicable to the backward comparison method used in this section for BPM validation. Therefore, the forward comparison (Test \#2) is used in Section 2.2.2 to validate the BPM.

\subsubsection{Forward predictions and comparisons}

Table 1: BPM results and its forward comparisons of superstructure in Bridge\#0312xxx1 (Test\#2)

\begin{tabular}{cc}
\hline $\begin{array}{c}\text { Bridge } \\
\text { Component }\end{array}$ & $\begin{array}{c}\text { Average prediction } \\
\text { difference (\%) }\end{array}$ \\
Deck & 3.20 \\
Superstructure & 3.10 \\
Substructure & 3.20 \\
\hline
\end{tabular}

The BPM training inputs for forward prediction employs the results of Test \#1 (from 1968 to 1994). The BPM generates the future direction of bridge condition ratings between 1996 and 2004. Table 1 shows average prediction difference of three bridge components. The average difference between the actual and generated data represents satisfactory results with $3.20 \%$ for the deck, $3.10 \%$ for the superstructure and $3.20 \%$ for the substructure. Therefore, the forward comparison method constructed in this section can be used to validate the BPM results using the actual BMS condition rating inputs.

\section{Case studies}

The verification of the BPM has been carried out via nine case studies i.e. 9 typical bridge elements from 7 bridges owned by the Roads and Transport Authority of New South Wales (RTA NSW), Australia under three different condition state scales (i.e. 3, 4 and 5-CS) using historical bridge 

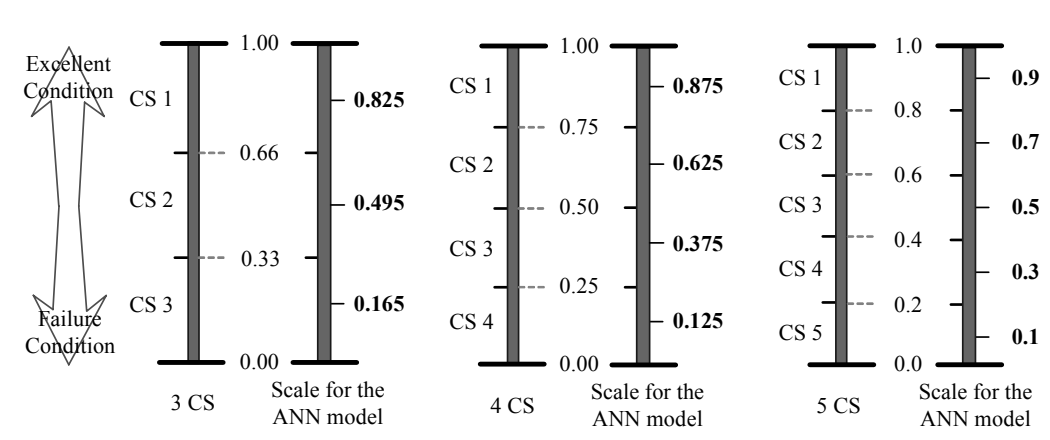

inspection records provided by

the RTA. The details of nine

bridge elements are presented in

Table 3 together with the summary of results. The three

different condition rating scales

for nine elements are illustrated

in Fig.2. The corresponding

years of non-bridge factors

including climates and

population growth for these case

Fig.2: Scales of bridge Condition States (CSs) for the BPM

studies are provided by the

Australian Government Bureau

of Meteorology and the Australian Bureau of Statistics (ABS). Most given bridges were built during the 1960s and 1970s with the approximate average life cycle of 40 years. The bridge element types in these case studies are defined in the RTA bridge inspection procedure manual [16]. The number of bridge element-level inspection datasets obtained for the BPM is mostly between 4 and 6 records for the 10 to 12 years of historical bridge element condition ratings. The 9 typical bridge elements are modelled to demonstrate the capability of the BPM.

\subsection{Neural-network modelling}

The BPM for these case studies has a single-layer feed-forward back-propagation neural network model. The specifications of the inputs, outputs and process functions for the BPM are described in Table 2. The input layer has seven factors, including five variables for climates and two variables Table 2: Components of BPM for case studies

\begin{tabular}{ll}
\hline Training Algorithm & Back-propagation feed-forward \\
Transfer Function & Log-sigmoid function \\
Input & Climates (5 factors) \\
& Population growth (2 factors) \\
Output & Bridge condition ratings (1 output) \\
\hline
\end{tabular}
for the population growth. These supplementary historical records enhance the lack of trends in the existing condition ratings so that reliable correlations among them can be obtained using the ANNbased BPM. The ANN training input is an $m$ by $n$ matrix where $m$ is the number of non-bridge factors (seven factors). The training output is formed correspondingly with the array of the training input. It also consists of an $m$ by matrix where $m$ is the number of inspections and $\mathrm{n}$ is the number of condition states (3CSs, 4CSs, and 5CSs). The compositions of the ANN testing input and output have an identical structure with the training datasets. The only difference is the size of matrix. This is because the number of years for predictions in the past time period is much greater than the number of years for condition rating prediction in the training stage.

In general, the size of the ANN training datasets is much greater than that of the testing input to establish their correlation. However, the available number of years for bridge element condition rating datasets for a training stage in this particular study is very limited. That is the major reason why the non-bridge factors are employed as supportive datasets for a limited amount of element condition ratings to generate missing years' condition ratings in the testing stage.

The outcomes from the BPM are the results of missing years' bridge element condition ratings. The number of prediction results in each year is 66 which is the combined number of learning rates (lr: $0.0-0.5$ ) and momentum coefficients (mc: $0.0-1.0)$ in the feed-forward back-propagation parameters whereas the latter only considers the optimal ANN parameters.

\subsection{BPM using typical bridge elements}

Among the selected 9 typical bridge elements, the timeframe of only one element (code CDSL concrete deck and slab) is shown in Fig.3. The figure is showing the time (years) for: (a) the entire bridge life cycle; (b) available condition ratings; (c) BPM inputs; (d) generated historical condition ratings; (e) inputs for validation; (f) forward-prediction results; and (g) result comparisons of the forward-predictions with existing condition rating datasets. 


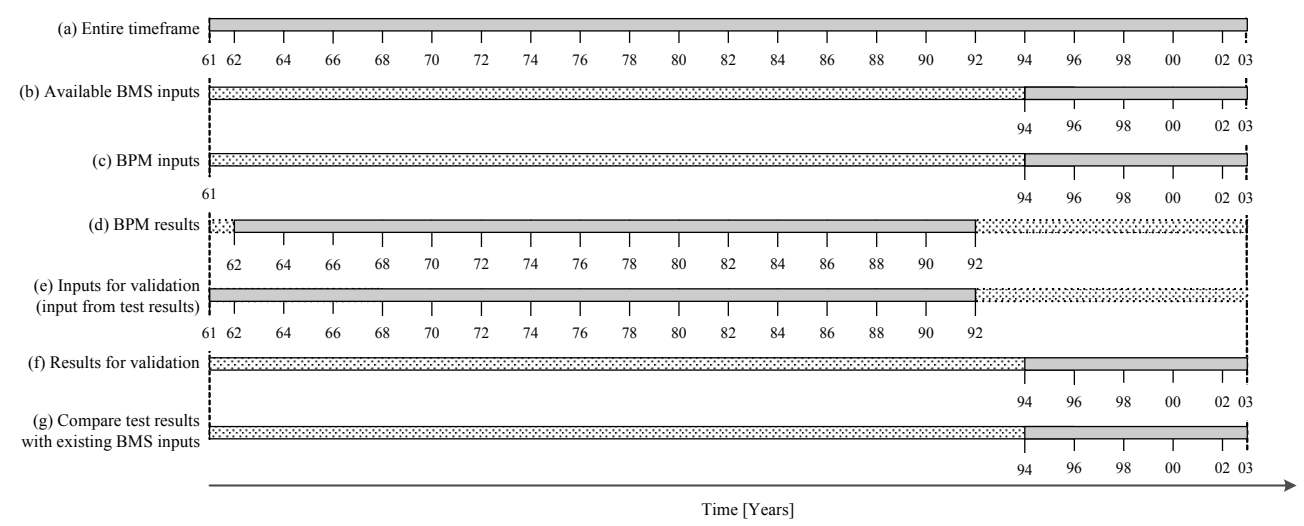

Fig.3: Timeframe for Element Code CDSL (Bridge \#5xx8)

\subsubsection{BPM using Element Code CDSL}

As shown in Fig.3 (b), the bridge element had 6 available inspection records for the years 19942003, which can be used to verify the proposed BPM. In this study, 16 records (i.e. 1962-1992 at 2year intervals) for the BMS condition rating inputs do not exist. The available 6 actual records for 1994-2003 plus the assumed "excellent" rating for year 1961, were used as input to the BPM to establish the relationships between the known non-bridge factors and the actual bridge condition ratings. These relationships were then used by the BPM in conjunction with the known non-bridge factors of 1962-1992, to produce the unavailable years of bridge element's condition ratings at 2 year intervals. This amounts to the generation of $72.72 \%$ of the total data (i.e. 16 unavailable records) using only $27.27 \%$ (i.e. 6 available records). In other words, the unavailable and available data ratio is 2.67 which is not uncommon for most major bridges in many a bridge agency.

The average quantity of each CS on this element between 1994 and 2003 is about $97.69 \%, 1.73 \%$ and $0.15 \%$ of the total element in CS1, CS2 and CS3 respectively. The six-inspection records use the ANN training inputs in the BPM, which generates historical condition ratings from 1962 to 1992 with two-year increments using three different element proportion rates. The BPM generates sixteen yearly historical condition ratings for the past thirty years from 1962 to 1992 for Element code CDSL. The average yearly predictions are plotted in Fig.4. Past condition ratings for about $97.97 \%$ of the total element as shown in Fig.4(a) are predicted. The others $(1.73 \%$ plus $0.15 \%$ of the total element) have major historical condition rating variances from those in the $1980 \mathrm{~s}$. Note in Fig. 5 that, the number of prediction results in each year is 66 , which is the combined number of learning rates and momentum coefficients in the neural network configuration.

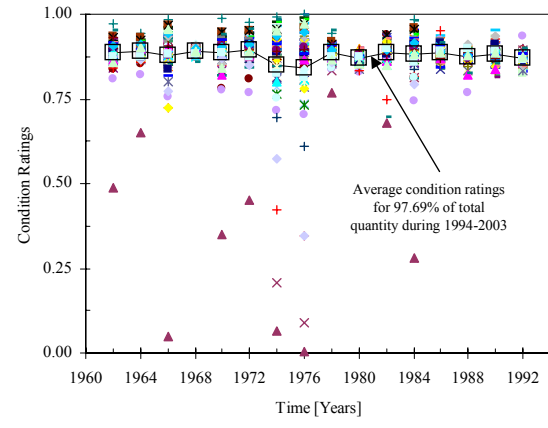

(a) BPM results: about $97.69 \%$ of total quantity

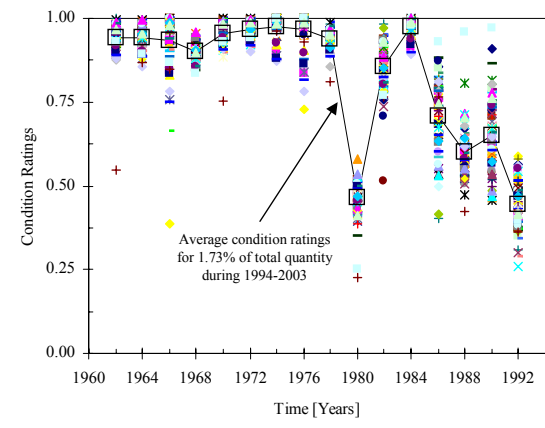

(b) BPM results: about $1.73 \%$ of total quantity

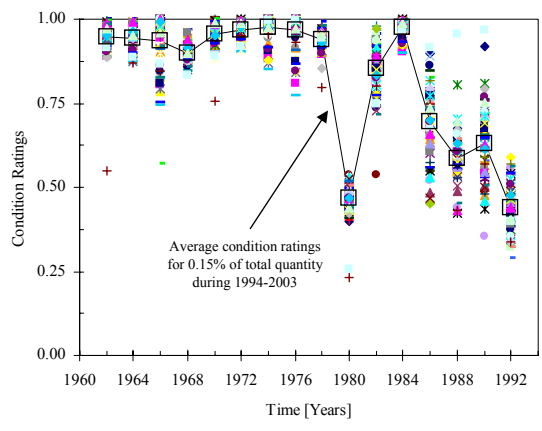

(c) BPM results: about $0.15 \%$ of the total quantity

Fig. 4: Backward prediction results for Element CDSL on Bridge \#5xx8

Post-calibration processes on BPM results are conducted to conform to the form of BMS' condition ratings. As a result, the generated historical condition ratings are detailed in Fig.5. 


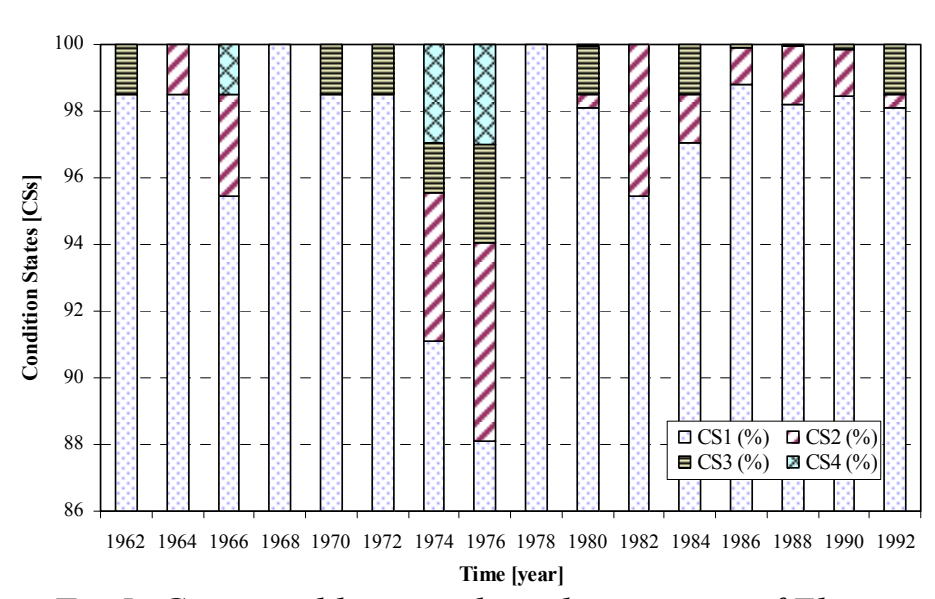

Fig.5: Generated historical condition states of Element CDSL for Bridge \# 5xx8 as BMS condition rating inputs
In order to validate the proposed BPM methodology, the "future" bridge condition ratings for the years 1994-2003 were predicted using the BPM generated condition ratings for the years 1962-1992. The neural network modelling for forward prediction has been setup to measure the BPM prediction accuracy. As illustrated in Fig.3(e), the BPM results from 1961 to 1992 including the assumed condition ratings in 1961, i.e. when the bridge was built and the condition rating of bridge element was at excellent level (CS1), are used as input datasets for this forward prediction neural network model. The forward-prediction neural network is trained by using the generated historical condition ratings with corresponding years of non-bridge factors to establish new correlation to predict six-inspection records from 1994 to 2003 . Results of this prediction can be directly compared with the existing condition rating datasets to measure prediction errors and to validate the BPM outcomes from 1962 to 1992.

Identical post-calibration processes in the BPM are required for the forward prediction results so that they can be compared with the existing condition ratings.

Fig. 6 shows the average prediction difference between each CS of the forward-prediction results and existing condition ratings. The overall prediction errors are shown to be less than the maximum error of $25 \%$. Therefore, the generated historical element condition rating using the 6 inspection records of element condition ratings on Element Code CDSL are reliable and hence can be stored into the BMS database as historical condition ratings.

\subsection{Summary of other typical elements}

Table 3: Prediction differences of the 9 typical bridge elements

\begin{tabular}{llrlrr}
\hline Element $\begin{array}{l}\text { code } \\
\text { Nascription }\end{array}$ & CSs & $\begin{array}{l}\text { Max. } \\
\text { errors }\end{array}$ & \multicolumn{2}{l}{ difference (\%) } \\
\cline { 5 - 6 } & & $\begin{array}{l}\text { Min. } \\
(\%)\end{array}$ & Max. \\
\hline JASS & Assembly Joint Seal & 3 & 33.33 & 1.69 & 2.5 \\
MMAS & Brick / Masonry / Reinforced Earth & 3 & & 3.95 & 18.61 \\
BELA & Elastomeric Bearing Pad & 3 & & 1.14 & 9.52 \\
\hline CDSL & Concrete Deck Slab & 4 & 25 & 0.71 & 4.18 \\
CPIL & Concrete Pile & 4 & & 11.03 & 12.21 \\
CPIR & Concrete Pier & 4 & & 1.63 & 4.47 \\
CPRG & Concrete Pre-tensioned Girder & 4 & & 0.73 & 1.56 \\
RMET & Metal Railing & 4 & & 5.81 & 23.89 \\
\hline LBGI & Steel(L) Beam / Girder & 5 & 20 & 1.14 & 4.4 \\
\hline
\end{tabular}

As shown in Table 3, the overall results from the case studies meet the pre-defined maximum prediction errors for the nine bridge elements. The maximum average yearly prediction errors are found to be $10.21 \%, 9.26 \%$ and $4.40 \%$ respectively for the three 
different condition states (CS's). These BPM results are considered satisfactory.

\section{Discussion \& Conclusion}

The methodology of the Backward Prediction Model (BPM) and the case studies are presented in this paper. Reliably predicting bridge elements' condition ratings from deterioration model are significant for BMS operations. This will in turn improve the accuracy of BMS outcomes based on which reliable maintenance strategies can be developed. The BPM described in this paper is the generation of unavailable historical data using very limited available condition ratings for long-term prediction of future bridge performance. This will be achieved by further development of the BPM. It should be noted that existing ANN-based "data-mining" techniques have been applied in medical, economics, engineering and IT fields. While capable of carrying out similar activities as the BPM, data-mining has had success only in cases where very small proportions of the datasets are missing - much smaller than are required to be generated for an effective BMS implementation.

The BPM uses non-bridge factors as supplementary historical data to overcome the lack of historical bridge data in terms of its quantity and patterns. The non-bridge factors including local climates, number of vehicles and population growth in the area surrounding the bridge are employed to help establish the correlations between the non-bridge factors and the lack of historical data patterns in the existing but inadequate bridge condition rating datasets.

The methodology of the BPM has been developed using bridge condition rating sample datasets made available by the Maryland Department of Transportation (Maryland DOT), and based on five existing condition rating datasets (or 26\% of the total record), the BPM is able to generate 14 missing datasets (or 74\%) for the intervening years when proper inspection records were missing. The average ratio of the generated and existing datasets is about 3 . The average prediction errors of the generated bridge condition ratings are between $6.7 \%-7.5 \%$ over a period of 20 years.

To further validate BPM methodology is conducted using 9 typical bridge elements from the Road and Traffic Authority of New South Wales (RTA NSW), Australia. The maximum yearly prediction errors of three different condition state scales $(3,4$, and 5-CS) are $18.61 \%, 23.89 \%$ and $4.40 \%$ respectively. These are satisfactory as compared to the maximum allowable prediction errors of $33.33 \%, 25 \%$ and $20 \%$ for 3-CS, 4-CS and 5-CS, respectively.

The outcome of the BPM has facilitated the establishment of a comprehensive condition rating database, which contains generated past condition ratings plus existing condition ratings. It can contribute to reliable predictions of bridge deterioration model because an entire life cycle of bridge's condition ratings contains more historical information than limited records.

The BPM is believed to be a useful methodology for an effective implementation of most commercial BMS packages and deserves wider application. In future, parametric studies should be conducted to determine the optimum numbers of non-bridge factors for different locations and types of bridges. This should lead to more effective use of the BPM by excluding the unnecessary nonbridge factors to yield a superior correlation between condition ratings and the well-chosen nonbridge factors.

\section{Acknowledgements}

The resources used for the present study were provided by Maryland State Department of Transportation, U.S. The authors wish to thank Messrs Earle Freedman and Matt Zulkowski from Maryland Department of Transportation for tracking our information requests. In particular, the authors would like to thank Prof. Waheed Uddin from The University of Mississippi for insightful comments and suggestions. The authors would also like to thank Mr. Perumynar Siva of the New South Wales Road and Traffic Authority, who has provided the important historical bridge datasets which enabled the case studies to be successfully completed.

\section{References}

[1] Hudson, W. R., Haas, R., and Uddin, W., "Infrastructure Management: Integrating Design, Construction, Maintenance, Rehabilitation and Renovation", McGraw-Hill, New York, 1997, $18-20$. 
[2] Godart, B., and Vassie, P. R., "Review of existing BMS and definition of inputs for the proposed BMS", BRIME Report Project PL97-2220, 1999, 18-22.

[3] Das, P.C., "Bridge Maintenance Management Objectives and Methodologies", Bridge Management 3: inspection, maintenance, assessment and repair, E\&FN Spon: London, 1996, $1-7$.

[4] DeStefano, P. D. and Grivas, D. A., "Method for estimating transition probability in bridge deterioration models", Journal of Infrastructure System, 4, 1998, 56-62.

[5] Lounis, Z. and Mirza, M. S., "Reliability-based service life prediction of deteriorating concrete structures", In the Proce. of 3rd Int. Conference on Concrete Under Severe Conditions, University of British Columbia, Canada. 2001.

[6] Sianipar, P. M. M. and Adams, T. M., "Fault-tree model of bridge element deterioration due to interaction", Journal of Infrastructure System, 3, 1997, 103-110.

[7] Hearn, G., "NDE in Bridge Management System", Eighth International Bridge Management Conference, 1999, C-1/1-13.

[8] Kawamura, K., and Miyamoto, A., "Condition state evaluation of existing reinforced concrete bridges using neuro-fuzzy hybird system”, Computer and Structures, 81, 2003, 1931-1940.

[9] Bolukbasi, M., Mohammadi, J., and Arditi, D., "Estimating the future condition of highway bridge components using national bridge inventory data", Practice Periodical on Structural Design and Construction, 9(1), 2004, 16-25.

[10] Johnson, B., and Nelson, F. J., "Project Selection Method Integrating BMS Data and Non deterioration Based Needs", Eighth International Bridge Management Conference, Denver, Colorado, 1999, I-2/1-9.

[11] DeStefano, P. D., Grivas, D. A., and Cornelius, S. A., "A reliability-based deterioration model for bridge maintenance planning", Infrastructure condition assessment: art, science, and practice, M. Saito, ed., ASCE, New York, 1997, 31-40.

[12] Abu-Tair, A. I., McParland, C., Lyness, J. F., and Nadjai, A., "Predictive models of deterioration rates of concrete bridges using the factor method based on historic inspection data", 9th International Conference on the Durability of Building Materials \& Components, Brisbane, Australia, 2002, 262.

[13] Tabachinick, B. G. and Fidell, L. S., "Using Multivariate Statistics", Allyn and Bacon, 2001.

[14] Mishalani, R. G., and McCord, M. R., "Infrastructure Condition Assessment, Deterioration Modeling, and Maintenance Decision Making: Methodological Advances and Practical Considerations", Journal of Infrastructure Systems, 12(3), 2006, 145-146.

[15] Lee, J.H., Sanmugarasa, K., Blumenstein, M. and Loo, Y.C., "Improving the reliability of a Bridge Management System (BMS) using an ANN-based Backward Prediction Model (BPM)", Journal of Automation in Construction (Accepted for the publication on 23/10/07).

[16] Road and Traffic Authority of New South Wales, "RTA Bridge Inspection Procedure", RTA NSW, 1999. 